\title{
COMPORTAMENTO DE CULTIVARES DE ARROZ IRRIGADO EM RELAÇÃO A FUNGOS MANCHADORES DE SEMENTES (')
}

\author{
JACIRO SOAVE $\left({ }^{2,7}\right)$, MARIA ANGÉLICA PIZZINATTO $\left({ }^{2,7}\right)$, \\ JOSÉ ALFREDO USBERTI FILHO $\left({ }^{3,7}\right)$, LUIZ ERNESTO AZZINI $\left({ }^{3,7}\right)$, \\ OCTÃVIO BENTO DE ALMEIDA CAMARGO $\left({ }^{4,7}\right)$, OMAR VILLELA $\left({ }^{5},{ }^{7}\right.$ ) \\ e PAULo Boller GaLlo $\left({ }^{6}, 7\right.$ )
}

\begin{abstract}
RESUMO
Em ensaios regionais instalados no ano agrícola de 1980/81, em Tremembé e Pindamonhangaba (SP), determinaram-se, em 37 cultivares de arroz irrigado, os fungos responsáveis por manchas em sementes de arroz e o comportamento desses cultivares em relação a cada fungo detectado. Amostras de sementes foram coletadas da produção total de cada parcela experimental, procedendo-se à separação visual das sementes manchadas e sem manchas e, posteriormente, aos cálculos da porcentagem de sementes manchadas e da porcentagem de perda de peso devida às manchas. Testes de patologia de sementes detectaram a presença das seguintes espécies de fungos: $A l$. ternaria sp., Cephalosporium sp., Cladosporium spp., Corynespora sp., Curvularia spp., Epicoccum spp., Fusarium moniliforme, Helminthosporium oryzae, Phoma spp., Pyricularia oryzae, Rhizoctonia solani, Rhynchosporium oryzae, Trichoconiella padwickii, Trichoderma sp. e Trichothecium sp. Diferenças altamente significativas entre o número de sementes manchadas e sem manchas foram observadas para os fungos $H$. oryzae, Phoma spp., $R$. oryzae e $T$. padwickii nos dois locais, patógenos então
\end{abstract}

( $\left.{ }^{1}\right)$ Trabalho apresentado no VII Congresso Paulista de Fitopatologia, realizado em Botucatu (SP), 23-25 de janeiro de 1984. Pesquisa parcialmente subvencionada pela EMBRAPA. Recebido para publicą̧ăo em 31 de maio de 1984.

(2) Seçāo de Microbiologia Fitotécnica, Instituto Agronômico (IAC), Caixa Postal 28, 13100 Campinas, SP.

( $\left.{ }^{3}\right)$ Seção de Genética, IAC.

$\left({ }_{5}^{4}\right)$ Seção de Arroz e Cereais de Inverno, IAC.

$\left.{ }^{5}\right)$ Estaçăo Experimental de Pindamonhangaba, IAC.

( $\left.{ }^{6}\right)$ Estação Experimental de Mococa, IAC.

( ${ }^{7}$ ) Com bolsa de suplementação do CNPq. 
considerados os principais responsáveis pela manchas de sementes observadas. A análise conjunta das duas localidades para cada parâmetro revelou 32 cultivares com menor porcentagem de sementes manchadas do que o controle comercial, quatro cultivares com menor porcentagem de perđa de peso devida às manchas, 24 com menor porcentagem de sementes com H.oryzae, seis com menor porcentagem de sementes com Phoma spp., todos os cultivares com menor porcentagem de sementes com R.oryzae e onze com menor porcentagem de sementes com T.padwickii do que o controle comercial. Considerando todos os parâmetros avaliados na análise conjunta e obtidos para as duas localidades, concluiu-se que os cultivares PI-1291, PI-1356, PI-1377, IR-1544, P2-48, P2-S2, P2-S1-78, L-17-72 e GI-6904-6 apresentaram melhor comportamento que os demais, pois foram superiores ou iguais ao controle em todos os parâmetros analisados.

Termos de indexação: sementes de arroz; resistência genética; patologia de sementes.

\section{INTRODUÇÃO}

O Instituto Agronômico de Campinas vem recebendo freqüentes consultas sobre manchas de sementes de arroz, mostrando sua crescente incidência em culturas comerciais de arroz, tanto de sequeiro como irrigado no Estado de São Paulo. O mesmo problema vem ocorrendo na região do Brasil Central, em lavouras de cerrado, conforme URBEN \& WETZEL (1980).

Esse problema vem ocorrendo há muito tempo em outros países, como Argentina, Guiana, Panamá, Costa Rica e Porto Rico (TAUBENHAUS et alii, 1935), e já vem sendo estudado nos Estados Unidos onde, segundo DOUGLAS \& TULLIS (1950), a perda anual em Arkansas, Lousiânia e Texás, no período 1930-1938, foi cerca de 463:000 dólares.

O complexo denominado manchas de sementes de arroz pode ser causado por grande número de fungos e, conforme a sintomatologia, recebe um nome especifico, embora nunca tenha sido determinada a presença de um único fungo causando a desordem. Assim, TISDALE (1922), pesquisando o "stack-burn" do arroz, encontrou sete gêneros de fungos nas sementes: Helminthosporium, Pyricularia, Epicoccum, Penicillium, Aspergillus, Fusarium e Rhizoctonia. TAUBENHAUS et alii (1935), estudando a doença conhecida como grãos negros ("black kernel"), constataram a presença de onze gêneros de fungos, na seguinte ordem de prevalência: Curvularia, Helminthosporium, Trichoconis, Basisporum, Fusarium, Cephalotecium, Rhizoctonia, Chaetomium, Alternaria, Epicoccum e Phoma. TULLIS (1936) detectou os seguintes gêneros de fungos em sementes de arroz manchadas: Curvularia, Fusarium, Trichoconis, Helminthosporium, Phoma, Alternaria, Cladosporium, Nigrospora, Epicoccum e Helicoceras.

Helminthosporium oryzae foi considerado um importante fungo causador de manchas em sementes por SCHROEDER (1964) e FAZLI \& SCHROEDER (1966). 
GUTIERREZ (1960), na Costa Risca, constatou, pela primeira vez, que Rhynchosporium oryzae pode causar considerável esterilidade, deformação de flores e descoloração das glumas da cariopse.

Além de fungos, o percevejo Oebalus pugnax (F.) pode causar manchas em grãos de arroz, segundo Douglas, citado por ATKINS (1974).

Do florescimento à maturação, as panfculas de arroz podem ser atacadas por vários fungos e, como conseqũência, ocorrer má granação ou manchas nos grãos, variando de leve descoloração até grãos completamente manchados e leves, que se perdem na operação de colheita, afetando a produção. Muitos grãos atacados são gessados e se quebram no beneficiamento, cujo rendimento é afetado. Por causa dessas perdas e do mau aspecto da produção, as manchas de sementes reduzem o preço do produto, diminuindo a renda do produtor.

No Brasil, tanto em culturas de arroz irrigado do Rio Grande do Sul, (RIBEIRO, 1979, e RIBEIRO \& MARIOT, 1974), como de arroz de sequeiro das regióes Sudoeste, Centro e Centro-Leste do País (URBEN \& WETZEL, 1980; LEÃO et alii, 1979, e LASCA et alii, 1979), foram constatados basicamente os mesmos gêneros de fungos citados como manchadores de sementes, com exceção de Rhynchosporium oryzae.

RIBEIRO (1979) sugere, como controle das manchas de sementes de arroz, fungicidas de amplo espectro de ação, semeadura em época normal e cultivares mais toleran tes a esses fungos.

Em 1980, começaram os estudos no Instituto Agronômico de Campinas, utilizando-se testes de sanidade de sementes de arroz para detectar os fungos prováveis manchadores de sementes e pesquisar o comportamento de cultivares quanto à incidência desses fungos, visando à seleção de cultivares de arroz menos suscetíveis aos agentes causadores de manchas e com menor porcentagem de sementes manchadas.

\section{MATERIAL E MÉTODOS}

Em dois munićpios do Vale do Pará́ba, Tremembé e Pindamonhangaba (SP), foram plantados experimentos com 37 cultivares de arroz irrigado, com delineamento experimental em blocos ao acaso e três repetições, atuando como controle comercial o 'IAC-899'. As parcelas foram constituídas por cinco linhas de $5 \mathrm{~m}$ de comprimento, espaçadas de $30 \mathrm{~cm}$. Foi utilizada adubação $10-60-30 \mathrm{~kg}$ de $\mathrm{N}, \mathrm{P}_{2} \mathrm{O}_{5}$ e $\mathrm{K}_{2} \mathrm{O}$ por hectare, mais $50 \mathrm{~kg}$ de $\mathrm{N}$ por hectare aplicados fracionados, em cobertura, aos 40 e 70 dias após a germinaçāo.

Além do potencial produtivo dos diversos germoplasmas de arroz 
em teste, visou-se também à avaliação do material quanto à tolerância aos prejuízos causados por manchas de grãos.

As três linhas centrais de cada parcela foram colhidas em conjunto, sendo enviada ao laboratório uma amostra de 200 gramas de sementes tiradas de cada parcela.

De cada amostra, foram tiradas três àmostras de trabalho de $5 \mathrm{~g}$, contando-se seu número de sementes manchadas e sem manchas. As sementes manchadas e as sem manchas de cada amostra foram pesadas separadamente. A partir desses dados, foram calculadas a porcentagem de sementes manchadas (SM) e a porcentagem de perda de peso devida às manchas pelas formulas:

onde:

$$
\% \mathrm{SM}=\frac{\mathrm{M}}{\mathrm{T}} \times 100
$$

$\% \mathrm{SM}=$ porcentagem de sementes manchadas;

$\mathrm{T}=$ peso total da amostra $(5 \mathrm{~g})$;

$\mathbf{M}=$ peso de sementes manchadas da amostra,

e

onde:

$$
\% \mathrm{PPDM}=\frac{\mathrm{PS}-\mathrm{PM}}{\mathrm{PS}} \times 100
$$

$\% \mathrm{PPDM}=$ porcentagem de perda de peso devida às manchas;

PS = peso de cem sementes sem mancha;

$\mathbf{P M}=$ peso de cem sementes manchadas.

Os valores \% SM e \% PPDM foram transformados em arco seno $\sqrt{\%}$ e analisados estatisticamente.

Cinqüenta sementes manchadas e cinqüenta sem manchas de cada parcela foram analisadas separadamente, pelo método padrão do papel de filtro, seguindo método recomendado por INTERNATIONAL . . . (1966), AULAKH et alii (1974), MATHUR \& NEERGAARD (1972), e KANG et alii (1972).

Cada placa de Petri de plástico transparente continha 25 sementes sem pré-tratamento, que foram incubadas a $22^{\circ} \mathrm{C}$ e expostas ao regime alternado de luz, com doze horas em luz negra e doze horas no escuro, durante sete dias, quando então foram examinadas com auxílio de um microscópio estereoscópico ( 6 a $50 \mathrm{X}$ ) e de um microscópio óptico comum.

$O$ número de sementes de cada parcela com cada fungo foi anotado e os resultados, após transformação em $\sqrt{x+0,5}$, foram analisados estatisticamente. Somente os fungos que apresentaram diferença estatística ao nível de $1 \%$ entre o número de sementes com e sem manchas foram analisados 
para detectar diferenças entre cultivares quanto ao numero de sementes com a presença desse determinado fungo.

Os resultados médios das porcentagens de sementes manchadas, de perda de peso devida às manchas e de sementes com Helminthosporium oryzae, Phoma spp., Rhynchosporium oryzae e Trichoconiella padwickii dos 37 cultivares foram contrastados pelo teste de Dunnett a 5\% para comparação dos cultivares com o controle comercial.

\section{RESULTADOS}

Foram constatados, nas duas localidades, os seguintes fungos nas sementes: Alternaria sp., Cephalosporium sp., Cladosporium spp., Corynespora sp., Curvularia spp., Epicoccum spp., Fusarium moniliforme, Helminthosporium oryzae, Phoma spp., Pyricularia oryzae, Rhizoctonia solani, Rhynchosporium oryzae, Trichoconiella padwickii, Trichoderma sp. e Trichothecium sp.

A análise da variância do número de sementes com Helminthosporium oryzae, Phoma spp., Rhynchosporium oryzae e Trichoconiella padwickii revelou diferenças altamente significativas entre os dois tipos de sementes, manchadas ou não, mostrando-se esse fungos responsáveis pelas manchas de sementes nas duas localidades. Os demais fungos detectados não foram considerados responsáveis pelas manchas das sementes. De fato, ocorreram em freqüência muito baixa, e as análises das variâncias deram coeficientes de variaçđo muito elevados, não permitindo revelar diferenças entre as sementes manchadas e as sem manchas.

No quadro 1 são apresentados os resultados médios das porcentagens de sementes manchadas, de perda de peso devida às manchas e de sementes com Helminthosporium oryzae, Phoma spp., Rhynchosporium oryzae e Trichoconiella padwickii, obtidos pela análise das sementes dos 37 cultivares de arroz do ensaio de Tremembé.

Nesse local, todos os cultivares apresentaram porcentagem de sementes manchadas menor que o controle comercial IAC-899, exceto PI-1291, PI-1332, P2-S2 e GI-6902. Os cultivares IAC-4440, IR-3518, IAC-1 278, PI-1291, PI-1332, PI-1356, PI-1377, IR-1544, P2-S1-78, GI-74-27 e GI-6904-6 apresentaram menor porcentagem de perda de peso devida às manchas de sementes que o controle.

A maioria dos cultivares apresentou porcentagem de sementes com Helminthosporium oryzae menor que o controle comercial, exceto IAC-4440, BR-541, IAC-1 278, PI-1377, IR-1 544, L-8-38, L-7-33, L-8-36, L-1-43, GI-6904-4 e GI-74-27. Os cultivares P2-S2, GI-74-27 e GI-74-29 mostraram 


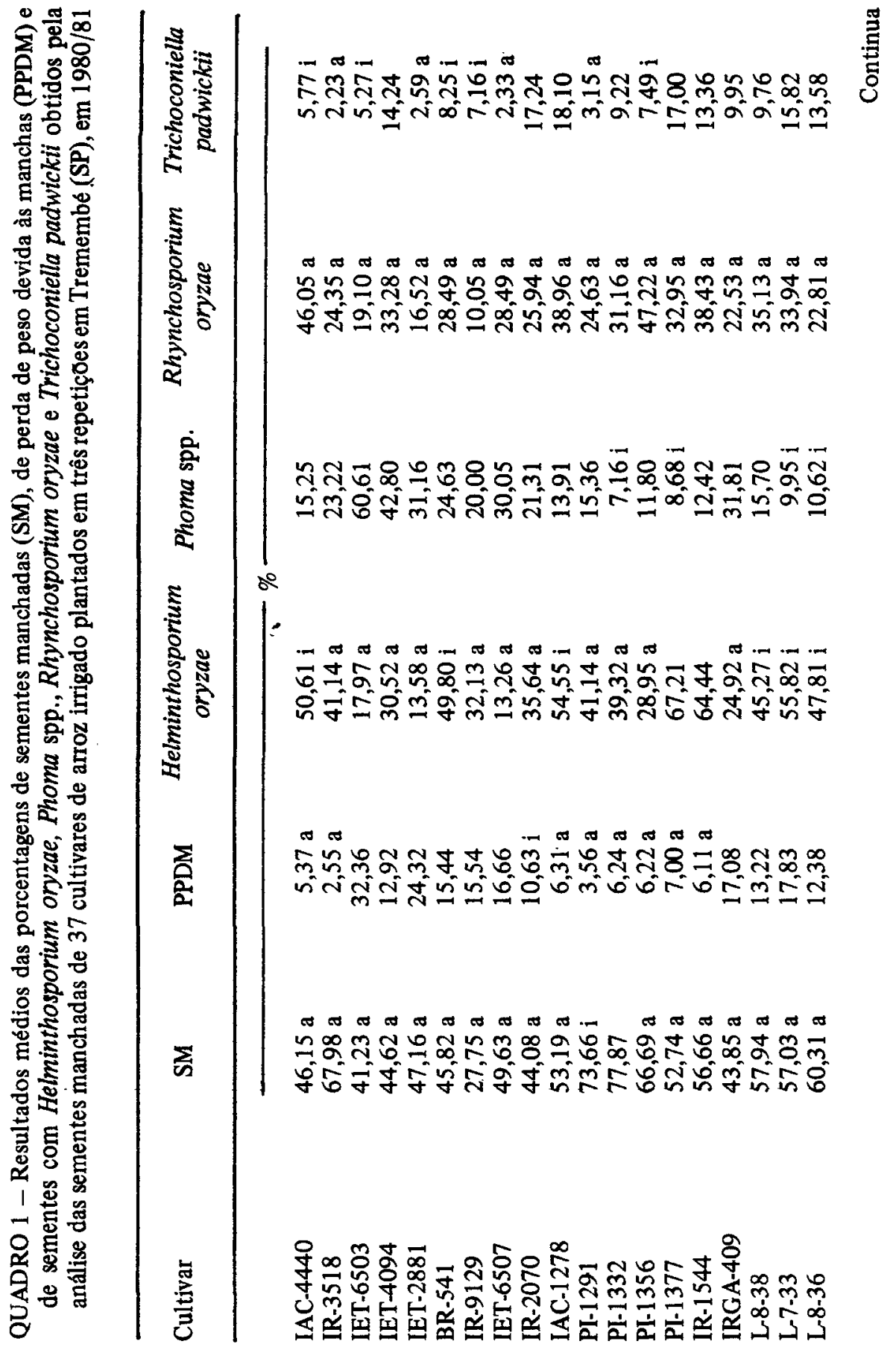




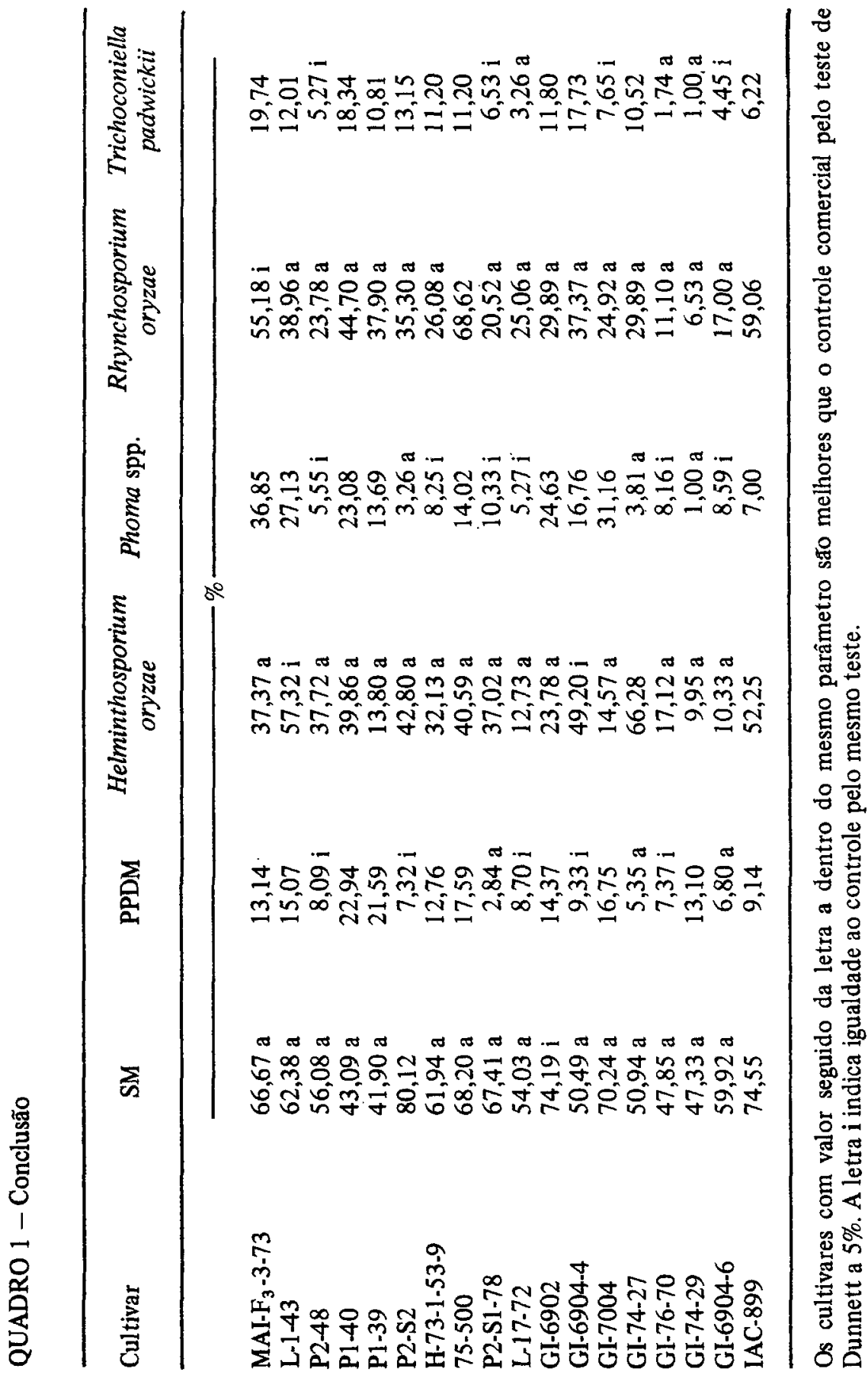


menor porcentagem de sementes com Phoma spp. que o controle. Todos os cultivares apresentaram porcentagem de sementes com Rhynchosporium

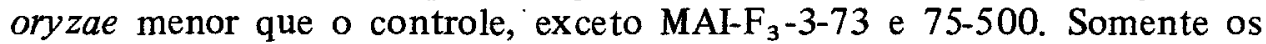
cultivares IR-3518, IET-2881, IET-6507, PI-1291, L-17-72, GI-76-70 e GI-74-29 apresentaram porcentagem de sementes com Trichoconiella padwickii menor que o controle IAC-899.

Levando em conta todos os parâmetros analisados no ensaio de Tremembé, pode-se concluir que os cultivares P2-48, P2-S1-78, L-17-72, GI-76-70 e GI-6904-6 apresentaram melhor comportamento que os demais, pois foram superiores ou iguais ao controle em todos os parâmetros avaliados.

No quadro 2 são apresentados os resultados médios da porcentagem de sementes manchadas, de perda de peso devida às manchas das sementes e de sementes com Helminthosporium oryzae, Phoma spp., Rhynchosporium oryzae e Trichoconiella padwickii, obtidos pela análise das sementes dos 37 cultivares de arroz irrigado do ensaio de Pindamonhangaba.

Todos os cultivares apresentaram porcentagem de sementes manchadas menor que o controle comercial, com exceção do PI-1291, PI-1332, PI-1356 e GI-6902, enquanto somente os cultivares IR-3518 e PI-1291 apresentaram menor porcentagem de perda de peso devida às manchas de sementes que o 'IAC-899'.

A maioria dos cultivares apresentou porcentagem de sementes com Helminthosporium oryzae menor que o controle comercial, exceto IR-3518, IET-6503, IET-4094, IET-2881, BR-541, IAC-1278, L-1-43, P2-48, P1-40, H-73-1-53-9, GI-6902, GI-7427, GI-76-70 e GI-7429. A maioria também apresentou porcentagem de sementes com Phoma spp. menor que o controle, com exceção do IAC-4440, IR-3518, IET-6503, IET-2881, BR-541, IR-9129, IAC-1 278, PI-1377, IRGA-409, P1-40, GI-6902, GI-7004, GI-76-70 e GI-74-29. Todos eles, sem exceção, apresentaram porcentagem de sementes com Rhynchosporium oryzae menor que o controle comercial, enquanto os cultivares IAC-4440, IR-3518, BR-541, IR-9129, PI-1 291, PI-1332, PI-1356, PI-1377, IR-1544, IRGA-409, L-8-38, L-7-33, L-8-36, P1-39, 75-500, P2-S1-78, GI-7004, GI-74-27, GI-76-70 e GI-7429 apresentaram porcentagem de sementes com Trichoconiella padwickii menor que seu controle.

Considerando todos os parâmetros analisados no ensaio de Pindamonhangaba pode-se concluir que os cultivares IAC-4440, IAC-1278, PI-1291, PI-1377, IRGA-409, P2-S1-78 e L-17-72 apresentaram melhor comportamento que os demais, em virtude de terem sido superiores ou iguais ao controle em todos os parâmetros.

No quadro 3 são apresentados os resultados médios da análise conjunta de Tremembé e Pindamonhangaba, da porcentagem de manchas das 


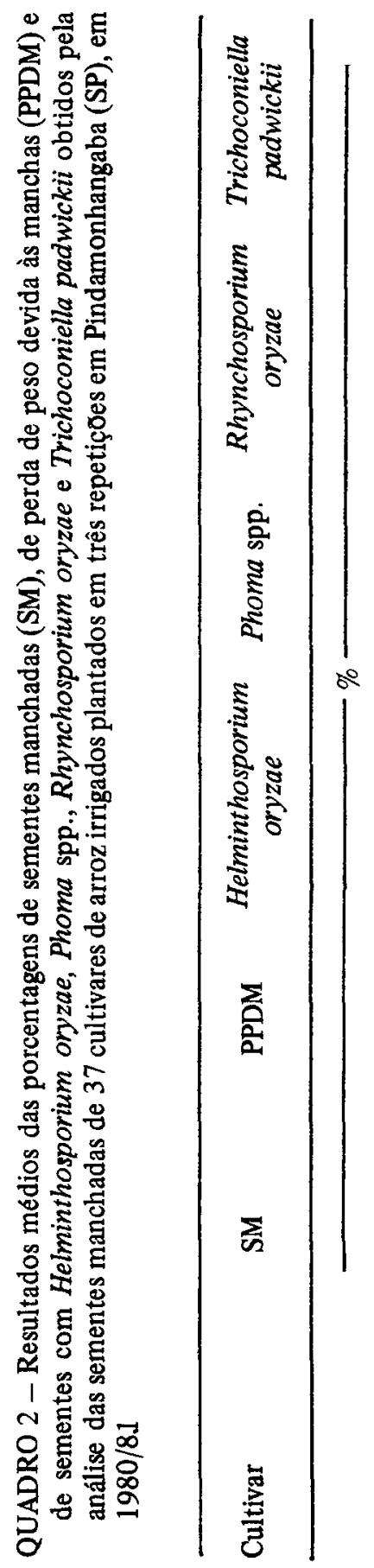

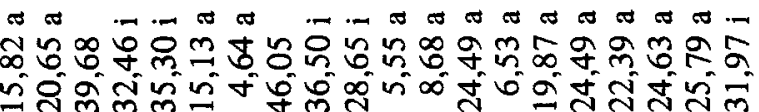

苞

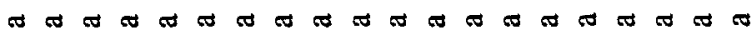

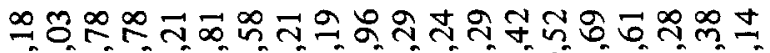

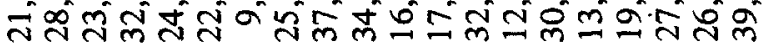

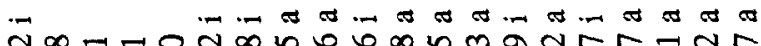

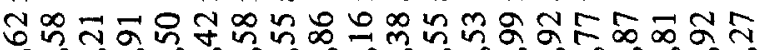

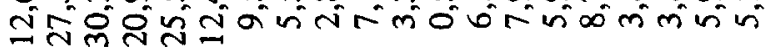

๘-1.

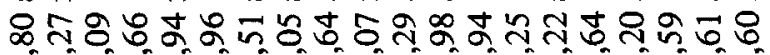

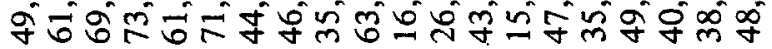

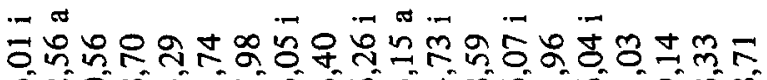
ơ 0 m

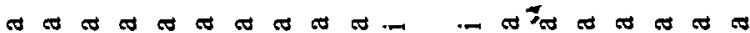

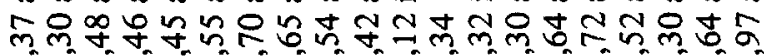
तीं

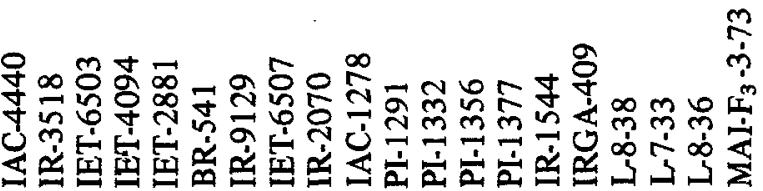




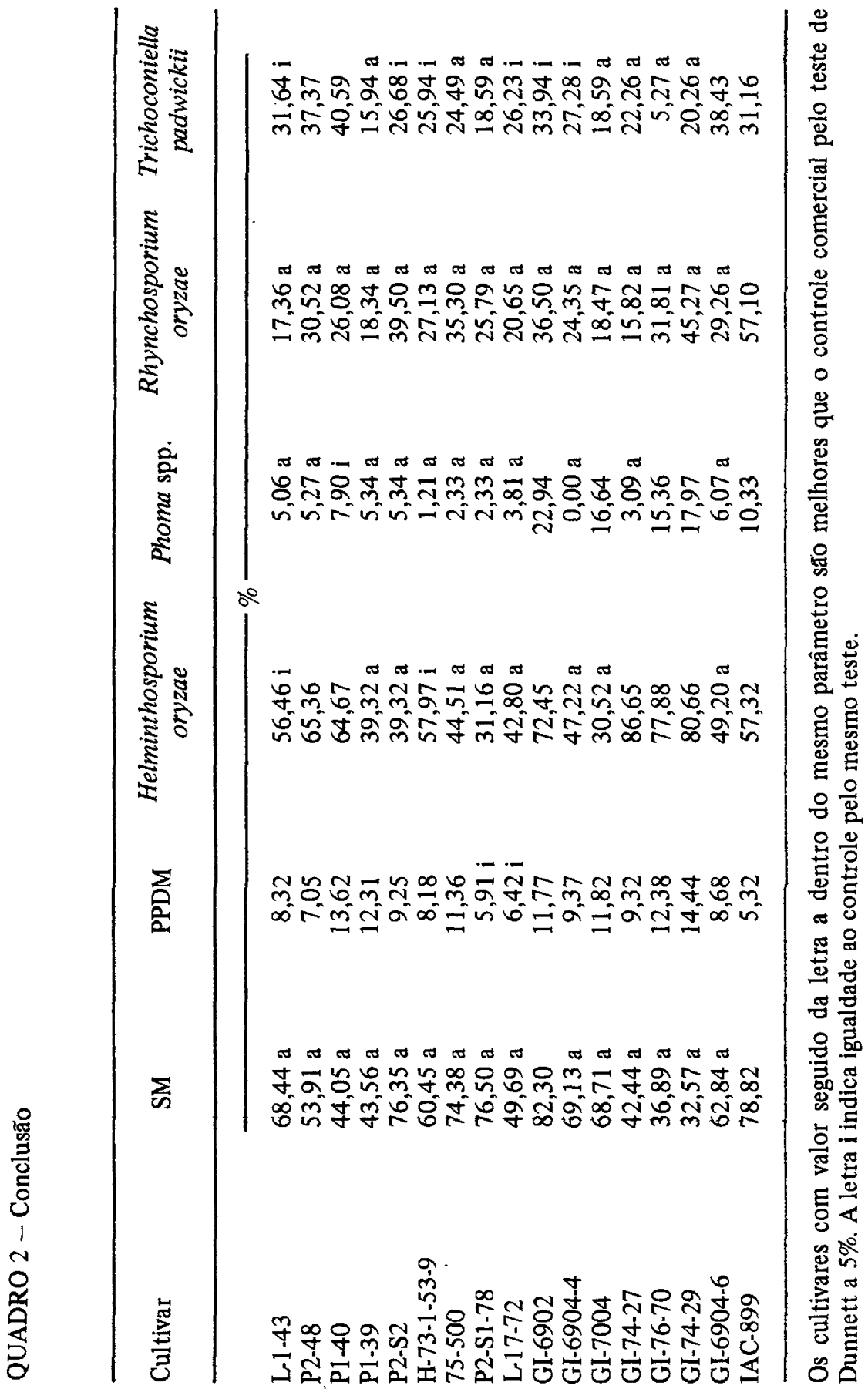




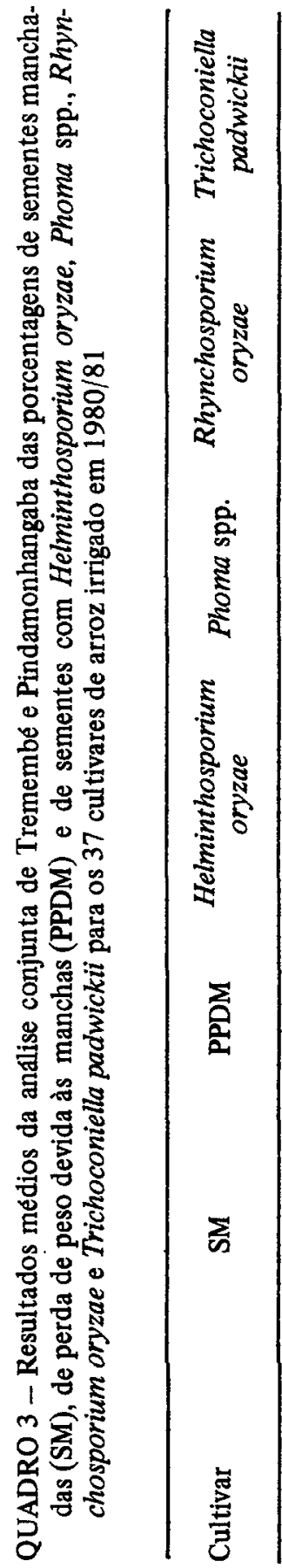

य. ํํㄱำ

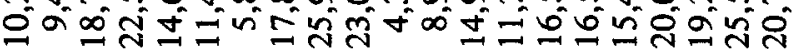

य ब 寸

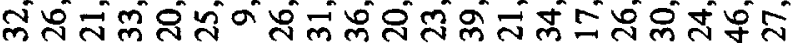

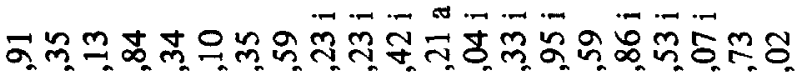

mn

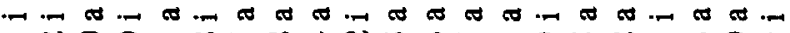

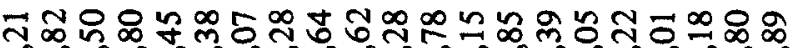
ही

-

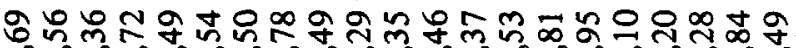

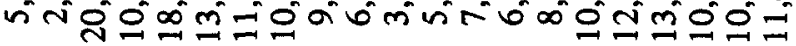

य 2 a 들 in in f f व

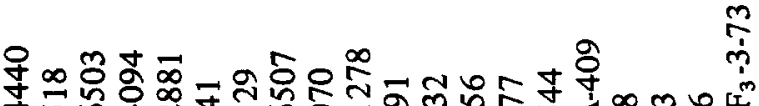

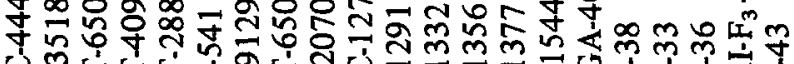

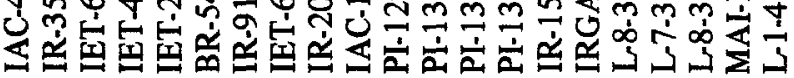




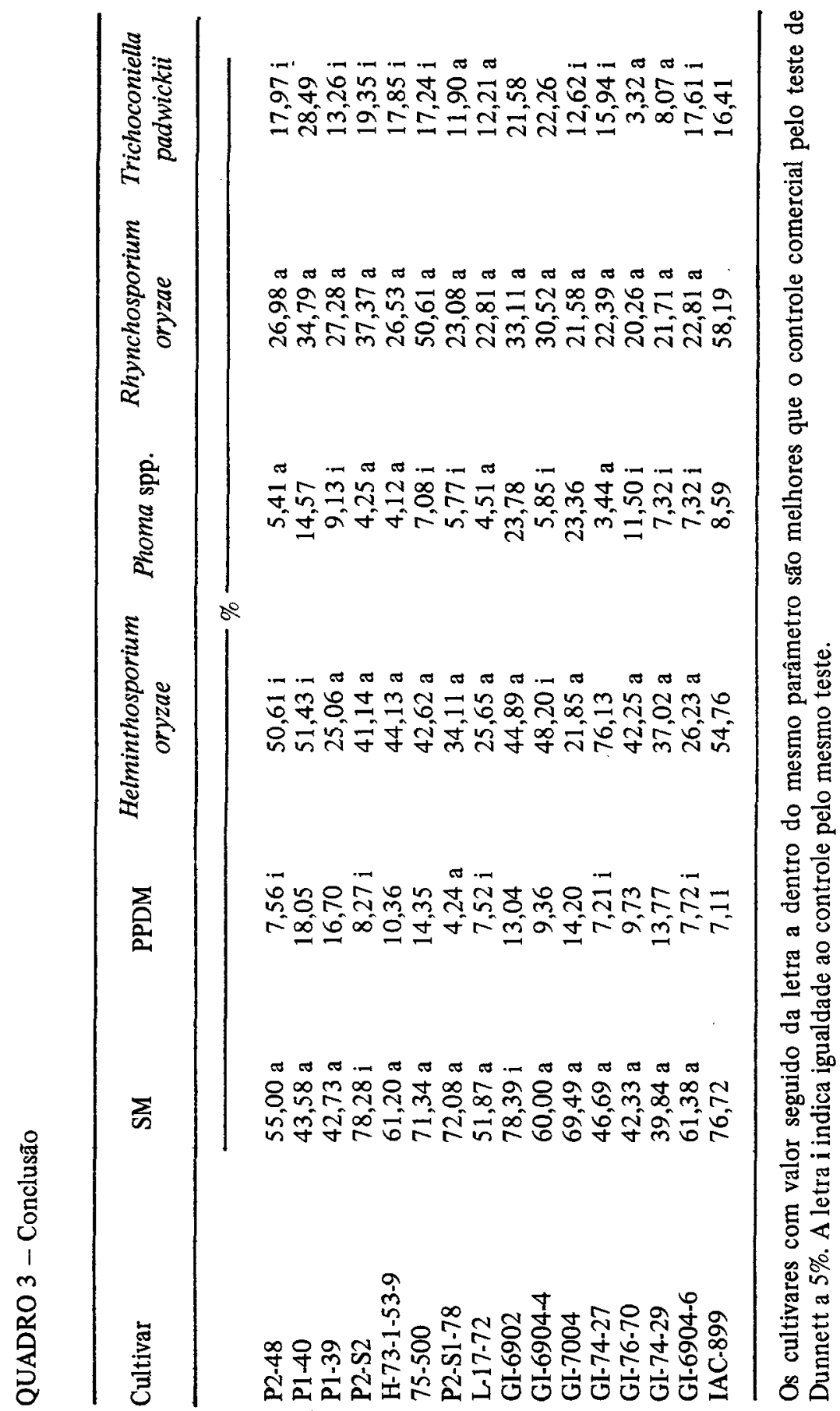


sementes, e das porcentagens de sementes com Helminthosporium oryzae, Phoma spp., Rhynchosporium oryzae e Trichoconiella padwickii, para os 37 cultivares de arroz irrigado.

A análise conjunta de cada parâmetro das duas localidades revelou que todos os cultivares, exceto PI-1291, PI-1332, P2-S2 e GI-6902, apresentaram porcentagem de sementes manchadas menor que o controle comercial, enquanto apenas os cultivares IR-3518, PI-1 291, PI-1332 e P2-S1-78 apresentaram porcentagem de perda de peso devida às manchas menor que o controle IAC-899.

A análise conjunta revelou ainda que a maioria dos cultivares apresentou porcentagem de sementes com Helminthosporium oryzae menor que o controle comercial, exceto IAC-4440, IR-3518, IET-4094, BR-541, IAC-1 278, IR-1544, L-7-33, L-1-43, P2-48, P1-40, GI-6904-4 e GI-74-27. Somente os cultivares PI-1332, P2-48, P2-S2, H-73-1-53-9, L-17-72 eGI-74-27 apresentaram porcentagem de sementes com Phoma spp. menor que o controle. Todos os cultivares, sem exceção, apresentaram porcentagem de sementes com Rhynchosporium oryzae menor que o controle comercial, enquanto os cultivares IAC-4440, IR-3518, BR-541, IR-9129, PI-1332, PI-1377, PI-1 291, P2-S.1-78, L-17-72, GI-76-70 e GI-7429 apresentaram porcentagem de sementes com Trichoconiella padwickii menor que o controle IAC-899 nas duas localidades.

Levando em consideração todos os parâmetros avaliados na análise conjunta das duas localidades, pode-se concluir que os cultivares PI-1291, PI-1 356, PI-1377, IR-1544, P2-48, P2-S2, P2-S1-78, L-1 7-72 e GI-6904-6 apresentaram melhor comportamento que os demais, em virtude de terem sido superiores ou iguais ao controle comercial IAC-899 em todos os parâmetros analisados.

\section{DISCUSSÃO E CONCLUSÕES}

Os fungos constatados nas sementes dos 37 cultivares de arroz irrigado, nas duas localidades do Estado de São Paulo, foram praticamente os mesmos já citados por pesquisadores de outros países (DOUGLAS \& TULLIS, 1950; TAUBENHAUS et alii, 1935; TISDALE, 1922; TULLIS, 1936).

No Brasil, Epicoccum sp., Trichoconis padwickii, Phoma spp., Helminthosporium oryzae, Pyricularia oryzae, Curvularia spp., Cladosporium spp., Trichoderma sp., Rhizoctonia solani, Alternaria sp. e Fusarium moniliforme, entre outros, já foram constatados por LEĀO et alii (1979), LASCA et alii (1979), RIBEIRO (1979), RIBEIRO \& MARIOT (1974) e URBEN \& WETZEL (1980). Apenas o fungo Rhynchosporium oryzae não havia sido 
constatado em sementes de arroz no Brasil ate 1980, tendo sido citado pela primeira vez somente na Costa Rica por GUTIERREZ (1960) como patógeno de semente de arroz.

Mediante a presente pesquisa, os fungos Helminthosporium oryzae, Phoma spp., Rhynchosporium oryzae e Trichoconiella padwickii podem ser considerados importantes manchadores de sementes, podendo concluir, também, que as manchas de sementes de arroz podem ser causadas por um ou mais patógenos, dependendo do local onde for cultivado. Isso mostra a necessidade de efetuar testes em diferentes localidades para seleção de cultivares menos suscetiveis a manchas de sementes.

LASCA et alii (1979) mostraram que em 1975 nao ocorreu $H$. oryzae em sementes do cultivar Pratão Precoce, mas foi encontrado em $80 \%$ das amostras do 'Batatais', enquanto em 1976 o patógeno apareceu em todas as amostras de 'Pratão Precoce' e ñ̃o nas de 'Batatais', o que demonstra que, além de testes em diferentes localidades, há necessidade de testes por mais de um ano, para se ter uma seleção mais segura de cultivares mais tolerantes aos fungos manchadores de sementes.

Os cultivares PH 1291, PI-1356, PI-1377, IR-1544, P2-S1-78, L-1 7-72, P2-48, P2-S2 e GI-69046 deverão ser selecionados como os melhores materiais, levando-se em conta todos os parâmetros analisados.

Da mesma forma, os cultivares IET-4094, MAI-F $3-3-73$, L-1-43, P1-40, GF6902 e GI-6904-4 se comportaram como os piores materiais para manchas de sementes, devendo ser eliminados dos testes.

\title{
SUMMARY
}

\section{PERFORMANCE OF LOWLAND RICE CULTIVARS TO SEED DISCOLORATION FUNGI}

\begin{abstract}
Aiming to detect rice seed discoloration fungi, the behavior of 37 lowland rice cultivars to each of them was determined in two field trials carried out with three replications in two sites of the State of Sáo Paulo (Tremembe and Pindamonhangaba), Brazil, in 1980/81. Seed samples were drawn from the whole yield of each experimental plot. Spotted and spotless seeds were visually separated and discolored seed (DS\%) and weight loss percentages (WL\%) calculated. From each treatment 50 spotted and 50 spotless seeds were plated separately in Petri dishes and incubated by the blotter method. The following fungi species were detected: Altemaria sp., Cephalosporium sp., Cladosporium spp., Corynespora sp., Curvularia spp., Epicoccum spp., Fusarium moniliforme, Helminthosporium oryzae, Phoma spp., Pyricularia oryzae, Rhizoctonia solani, Rhynchosporium oryzae, Trichoconielle padwickii, Trichoderma sp. e Trichothecium sp. In relation to the number of seeds infected with each fungus, highly significant statistical differences between spotted and spotless seeds were detected for $H$. oryzee, Phome spp., $R$. oryzae and $T$. padwickii. They were considered as causal agents of the spots. The statistical analysis of the data related to each parameter showed that a variable number of cultivars out performed the commercial control, as to each respective parameter studied: 32 as to DS\%; 4 as to WL\%; 24 as to $H$.
\end{abstract}


oryzae; 6 as to Phome spp.; all cultivars as to $R$. oryzae and 11 as to $P$. padwickii. The statistical analysis of all parameters in the two sites showed the cultivars PI-1291, PI-1356, PI-1377, IR-1544, P2-S1-78, L-17-72, P2-48, P2-S2 and GI-6904-6 as the best ones when compared with the commercial control IAC-899.

Index terms: rice seeds; genetic resistance; seed pathology.

\section{REFERÊNCIAS BIBLIOGRÁFICAS}

ATKINS, J. G. Rice disease of the Americas: a review of literature. Washington, U. S. Dept. of Agriculture, 1974. 106p. (Agriculture Handbook, 448)

AULAKH, K. S.; MATHUR, S. B. \& NEERGAARD, P. Seed health testing of rice and comparison of field incidence with laboratory counts of Drechslera oryzae and Pyricularia oryzae. Seed Science \& Technology, 2:393-398, 1974.

DOUGLAS, W. A. \& TULLIS, E. C. Insects and fungi as causes of pecky rice. Washington, U. S. Dept. of Agriculture, 1950. 20p. (Technical Bulletin, 1015)

FAZLI, S. F. I. \& SCHROEDER, H. W. Effect of kernel infection of rice by Helminthosporium oryzae on yield and quality. Phytopathology, 56: 1003-1005, 1966.

$\&$

Kernel infection of Bluebonnet 50 rice by Helminthosporium oryzae. Phytopathology, 56:507-509, 1966.

GUTIERREZ, L. C. de. Leaf scald of rice, Rhynchosporium oryzae in Costa Rica. Plant Disease Reporter, 44:294295, 1960.

INTERNATIONAL Rules for Seed Testing. Proceedings of the International Seed Tesiting Association, 31:1-152, 1966.

KANG, C. S. ; NEERGAARD, P. \& MATHUR, S. B. Seed health testing of rice. VI -Detection of seed-borne fungi on blotters under different incubation condition of light and temperature. Proceedings of the International Seed Testing Association, 37:731-740, 1972.

LASCA, C. C.; AMARAL, R. E. M. \& MALAVOLTA, V. M. A. Sanidade de sementes de arroz produzidas no Estado de São Paulo. In: REUNIÃO DE TÉCNICOS EM RIZICULTURA DO ESTADO DE SÃO PAULO, 1., Campinas, 1979. Anais. Campinas, CATI, 1979. p.123-124.

LEÃO, M. F.; LASCA, C. C. \& AMARAL, R. E. M. Ocorrência de fungos em sementes de arroz no Estado de Mato Grosso. In: REUNIÃO DE TÉCNICOS EM RIZICULTURA DO ESTADO DE SÃO PAULO, 1., Campinas, 1979. Anais. Campinas, CATI, 1979. p.107-114. 
MATHUR, S. B. \& NEERGAARD, P. Seed health testing of rice. IV -Effect of light and temperature on seed-borne fungi in the blotter test. Proceedings of the International Seed Testing Association, 37:723-730, 1972.

RIBEIRO, A. S. Doenças do arroz irrigado. Pelotas, EMBRAPA/UEPAE, 1979. 44p. (Circular Técnica, 3)

\& MARIOT, C. Condiçōes fitossanitárias das sementes de arroz no Rio Grande do Sul. Lavoura Arrozeira, Porto Alegre, 272:48-52, 1974.

SCHROEDER, H. W. Grain discoloration in Belle Patna rice. Plant Disease Reporter, 48:288-291, 1964.

TAUBENHAUS, J. J.; ALTSTATT, G. E. \& WYCHE, R. H. Black Kernel of Rice. In: ANNUAL REPORT, 48. Texas, Agricultural Experiment Station, 1935. p.94.

TISDALE, W. H. Seedling blight and stack-burn of rice and the hot-water seed treatment. Washington, U. S. Dept. of Agriculture, 1922. 11p. (Bulletin, 1116)

TULLIS, E. C. Fungi isolated from discolored rice kernels. Washington, U. S. Dept. of Agriculture, 1936. 11p. (Technical Bulletin, 540)

URBEN, A. F. \& WETZEL, M. M. V. S. Ocorrência, sobrevivência e controle de Phyllosticta oryzae em sementes de arroz produzidas nas condições dos cerrados. Fitopatologia Brasileira, Brasília, 5:1980. p.462. (Resumo) 